\title{
Regional patterns of 18F-Florbetaben uptake in Presenilin 1 mutation carriers
}

Roser Sala-Llonch $^{1 *}$, Neus Falgàs ${ }^{2}$, Beatriz Bosch ${ }^{2}$, Guadalupe Fernández-Villullas ${ }^{2}$, Mircea Balasa $^{2}$, Anna Antonell ${ }^{2}$, Andres Perissinotti ${ }^{3}$, Javier Pavía ${ }^{3,4}$, Francisco Campos ${ }^{3}$, Albert Lladó $^{2}$, Francisco Lomeña ${ }^{3}$, Raquel Sánchez-Valle ${ }^{2 *}$

${ }^{1}$ Dept. of Biomedicine, Faculty of Medicine, University of Barcelona, Barcelona, Spain

${ }^{2}$ Alzheimer's disease and other cognitive disorders. Neurology department. Hospital Clínic, Institut d'investigacions biomèdiques August Pi I Sunyer, University of Barcelona, Barcelona, Spain.

${ }^{3}$ Nuclear Medicine department, Hospital Clínic, Institut d'investigacions biomèdiques August Pi I Sunyer, Barcelona, Spain.

${ }^{4}$ Consorcio Centro de Investigación Biomédica en Red (CIBER) de Bioingeniería, Biomateriales y Nanomedicina (CIBER-BBN), Group of Biomedical Imaging, University of Barcelona, Barcelona, Spain.

* Corresponding authors

Keywords: Alzheimer's disease; biomarkers; familial Alzheimer's disease; presenilin1, florbetaben, PET, amyloid

\section{Corresponding authors:}

R. Sánchez-Valle

Alzheimer's Disease and Other Cognitive Disorders Unit, Department of Neurology,

Hospital Clínic, IDIBAPS, University of Barcelona.

Villarroel, 170. 08036. Barcelona. Spain.

Phone number: (+34)932275785. Fax: (+34)932275783.

E-mail address: rsanchez@clinic.cat

\section{R. Sala-Llonch}

Dept. of Biomedicine, Faculty of Medicine, University of Barcelona.

Casanova 143, 08036 Barcelona, Spain

Phone number : (+34) 934024516

E-mail address: roser.sala@ub.edu 


\begin{abstract}
Autosomal dominant Alzheimer's disease (ADAD) individuals present amyloid deposits before symptoms onset. We aimed to investigate efficacy and safety of $18 \mathrm{~F}$ - florbetaben (FBB) for assessing amyloid deposition in ADAD. We acquired FBB-PET and MRI of 25 individuals from PSEN1 families (NCT02362880). We studied individual uptake patterns, group differences, and correlation with estimated years to symptoms onset (EYO), as well as adverse events (AE). We found that asymptomatic carriers $(\mathrm{N}=14)$ showed increased FBB uptake across the cerebral cortex and in the caudate. FBB accumulation appeared more than 15 years before onset in precuneus and bankssts, among other regions, overlapping regions showing increased cortical thickness in the same subjects. FBB uptake correlated with EYO in several areas, especially the rostral anterior cingulate. Symptomatic carriers $(\mathrm{N}=7)$ had an elevated FBB uptake plateau. No AE were reported. Overall, we found progressive FBB uptake in ADAD starting two decades before symptoms. The rostral anterior cingulate is a candidate area to track $A \beta$ deposition in addition to the precuneus.
\end{abstract}




\section{INTRODUCTION}

Autosomal dominant Alzheimer's disease (ADAD), with almost 100\% penetrance, allows the study of the trajectories of different biomarkers and the evaluation of disease-modifying treatments at early or presymptomatic stages of the disease (Bateman et al., 2011). In addition, the relatively predictable age of onset in ADAD allows the calculation of the estimated years to symptom onset (EYO), as a proxy of the distance to symptoms onset for each asymptomatic mutation carrier. This facilitates the comparison between individuals with different mutations and the study of the progression of the disease even in cross-sectional samples. Using the EYO measure, research on ADAD, has revealed a temporal ordering of biomarkers indicating that the first pathological changes appear during the asymptomatic stage in target core regions and that these progressively expand towards new regions of the brain as the disease symptoms worsen. Brain amyloid- $\beta(A \beta)$ accumulation is one of these biomarkers, and two ongoing trials, the Dominantly Inherited Alzheimer Network Trials Unit (Mills et al., 2013) and the Alzheimer's Prevention Initiative (Carrillo et al., 2013), are already testing drugs that aim to limit the amyloid accumulation in the brain in both ADAD and sporadic Alzheimer's disease (AD).

Positron emission tomography (PET) imaging is a reliable tool to explore the patterns of amyloid deposition. Different amyloid-PET tracers available have demonstrated accurate correlation with post-mortem amyloid deposition. In ADAD, both the 11C-Pittsburgh compound B (PIB) (Bateman et al., 2012; Benzinger et al., 2013; Gordon et al., 2018; Quiroz et al., 2018; Rodriguez-Vieitez et al., 2016) and the 18F-Florbetapir (Fleisher et al., 2012) tracers have been used, detecting elevated amyloid uptake about 15 years before estimated symptom onset. In addition, some of the ADAD patients show relevant and early striatal amyloid tracer binding that is not frequent in sporadic AD (Benzinger et al., 2013). However, it has not yet been established whether different amyloid tracers bind to identical sites on $A \beta$ fibrils, offering the same ability to detect the regional $A \beta$ burden. Regional and temporal disparities in fibrillar $A \beta$ accumulation might have important implications for 
clinical trials. On the other hand, even if PIB-PET is considered the gold standard for amyloid tracers, its availability is more limited worldwide, given that it is a Carbon-tracer.

Florbetaben (FBB: trans-4-(N-methyl-amino)-4-\{2-[2-(2-[18F]fluoroethoxy) ethoxy]-ethoxy\} stilbene; Neuraceq ${ }^{\circledR}$ ) has high affinity and specificity for $A \beta$, lack of binding to Lewy bodies or neurofibrillary tangles in post-mortem tissue at low nanomolar concentrations, and excellent correlation with global PiB retention (Rowe et al., 2017). However, no studies have been published to date examining the spatial pattern and chronology of FBB binding in ADAD mutation carriers. In this study, we investigate for the first time the efficacy and safety of the $\beta$-amyloid tracer 18F-Florbetaben (FBB) for assessing the regional and chronological amyloid deposition pattern in ADAD due to PSEN1 mutations.

\section{MATERIAL AND METHODS}

\subsection{Participants}

Participants were recruited within the genetic counselling programme for familial dementias (PICOGEN) at the Hospital Clinic, Barcelona, Spain (Fortea et al., 2011). Participants were adult children ( $>18 \mathrm{y}$ ) of symptomatic ADAD patients with a mutation in the PSEN1 gene and who were either cognitively normal (Clinical Dementia Rating CDR=0) or had mild symptoms of cognitive decline (CDR 0.5 or 1). Exclusion Criteria were the presence of any condition precluding the completion of the study, any major medical disease, especially severe liver disease (AST /ALT $>5 \mathrm{x}$ ULN) or advanced renal insufficiency (creatinine $>2 \mathrm{x}$ ULN), current or previous history of alcohol abuse or epilepsy, known allergy to florbetaben or its constituents, pregnancy or breast feeding or planned pregnancy during the study period, and any medical or psychiatric disease other than ADAD, which could cause disturbance of brain function or cognition.

The study was approved by the Hospital Clinic ethics committee and all subjects gave written informed consent. According to the Spanish regulations related to the use of radiotracers in human subjects, the study was registered as a clinical trial (EudraCT No. 2014-000763-41, 
protocol code FBB-FAD-2014, ClinicalTrials.gov Identifier: NCT02362880) and approved by the Spanish Drugs and Medical Products Agency (AEMPS).

Participants were classified as asymptomatic if they had no cognitive complaints, their cognitive performance was normal, and they scored 0 on the CDR scale, or as symptomatic if their CDR score was $>0$ or if their cognitive performance was $\geq 1.5 \mathrm{SD}$ below the mean. In addition, according to their genetic status, participants were classified as mutation carriers (MC), either asymptomatic or symptomatic MC (AMC or SMC), and non-carriers or controls (CTR).

We calculated the EYO for AMC as the subject's age at the time of the study minus their parental age at symptoms onset. Further, we divided the AMC into two groups: stage I-AMC, those younger than 15 years from estimated symptoms onset, and stage II-AMC, older than 15 years from estimated symptoms onset. In SMC we calculated EYO as the subject's age at the time of the study minus their real age at symptoms onset.

\subsection{Safety measures}

Safety measures were recorded at the moment of the PET acquisition and after 24 hours. We recorded adverse events (AE) spontaneously reported by the participant, discovered as a result of general questioning by the study staff, or determined by physical examination, as well as findings of the medical and neurological examination and vital signs (blood pressure, heart rate, temperature).

\subsection{MRI acquisition}

Participants were examined on a 3T MRI scanner (Magnetom Trio Tim, Siemens Medical Systems, Germany). A high-resolution 3D structural data set (T1-weighted, MP-RAGE, TR = $2300 \mathrm{~ms}, \mathrm{TE}=2.98 \mathrm{~ms}$, voxel size $=1 \times 1 \times 1 \mathrm{~mm} ; \mathrm{FoV}=256 \mathrm{~mm}$ ) was acquired. Two subjects (one SMC and one AMC) were acquired in a different scanner with comparable sequences. 


\subsection{PET acquisition}

Each participant received a single intravenous bolus of approximately $300 \mathrm{MBq}(8.1 \mathrm{mCi})$ of [18F] Florbetaben, followed by a saline flush. Eighty minutes post-injection participants were scanned in a Siemens Biograph molecular-CT. The scanning included a low dose CT (50 mAs, $120 \mathrm{kV}$ ) for attenuation correction and a PET acquisition consisting in 4 5-minute dynamic 3D frames. PET images were corrected for attenuation, deathtime, dispersion and decay and reconstructed using an iterative algorithm (TRUEX+TOF; 8 iterations, 21 subsets, with a Gaussian post-filter FWWH of $3 \mathrm{~mm}$ ), resulting in the final sequence of whole brain images of 109 planes $(1.02 \times 1.02 \times 2.0 \mathrm{~mm})$. Summed images were subsequently created for quantification and analyses.

\subsection{Visual analysis of PET images}

The visual pattern of tracer retention was evaluated by 2 physicians (authors N.F. and F.L) blinded to the genetic and clinical status of the participants in the following areas: lateral temporal, frontal, parietal, posterior cingulate, precuneus, occipital, striatum and cerebellum. Interpretation of the images was made comparing activity of grey matter with activity in adjacent white matter. FBB uptake in each of these regions was classified as pathologic/positive or non-pathologic/normal. Furthermore, cortical retention was classified as focal retention if it was localized in a region or generalized uniform uptake if it covered several regions of the cortical mantle.

\subsection{MRI analysis}

Images were processed with Freesurfer (http://surfer.nmr.mgh.harvard.edu/), version 6.0. The Freesurfer stream includes cortical reconstruction and registration, subcortical segmentation (Fischl et al., 2002), delineation of pial and white matter surfaces, estimation of the cortical thickness (CTh), and parcellation of the cortical surface (Fischl et al., 2004). For whole-head segmentation of MRI data, we used an atlas specifically defined for PET analysis, consisting on 101 ROIs covering cortical and subcortical areas (Greve et al., 2016). 
We performed visual validation of the surface reconstruction and the segmentation results.

We calculated the mean CTh value within each cortical ROI of the atlas.

\subsection{Automated PET quantification and analysis}

Each individual PET scan was first registered to its corresponding MRI image using normalized mutual information (Greve and Fischl, 2009). All registrations were visually inspected.

We obtained the standardized uptake value ratio (SUVR) for each of the atlas ROIs, calculated as the mean uptake in the ROI divided by the uptake in the region of reference. We used the pons as a reference region according to previous studies ( $\mathrm{Su}$ et al., 2016). PET values were corrected for partial volume effects (PVE) using the geometrical transfer matrix (GTM) method (Greve et al., 2016). In order to reduce the number of measures, we calculated the bilateral mean (average between left and right) of the uptake in the main regions, resulting in 40 summary values per subject (6 subcortical and 34 cortical regions). We used a previously reported cutoff of 0.78 (Bullich et al., 2017) to calculate the number of subjects with high FBB uptake at each region within each group. However this threshold was obtained with results from a slightly different methodology based ROIs from the AAL atlas (Tzourio-Mazoyer, 2002). We used this threshold, as one of the most restrictive threshold reported in the mentioned study, in order to account for changes in the methodology (Freesurfer-based analysis with PVC versus AAL-based analysis with no PVC). In addition, to further explore these differences between methods, we obtained the quantification results with the AAL-based analysis. These methods, as well as the comparison between results from the two approaches are reported in Supplementary Material.

\subsection{Statistical analyses}

We compared the SUVR values between groups, using nonparametric tests in MATLAB. Specifically, we used Kruskal-Wallis and Mann-Whitney U for testing differences between groups of subjects. For each region we tested 4 hypotheses: (a) higher uptake in SMC 
compared with CTR; (b) higher uptake in AMC compared with CTR; (c) higher uptake in stage I-AMC compared with CTR; and (d) higher uptake in stage I-AMC compared with CTR. We also used Spearman correlations to assess the association between individual SUVR scores and EYO, calculated in all MC and in AMC and SMC subjects separately. As regards CTh, we also performed group comparisons and correlation with EYO in the 34 cortical bilateral parcellations. All tests were considered significant at $\mathrm{p}<0.05$ level. $\underline{\mathrm{We}}$ $\underline{\text { calculated differences after correction for multiple comparisons using the false discovery rate }}$ (FDR) correction, for each hypothesis and across all the regions evaluated, implemented in MATLAB (Benjamini \& Hochberg, 1995), with a false discovery rate of $\mathrm{q}<0.05$.

\section{RESULTS}

\subsection{Sample characteristics}

Twenty-five participants from ADAD families caused by 12 different PSEN1 mutations (E120K, H131R, V151M, H163R, S169P, L173F, G209E, K239N, L282R, L286P, G378R and I439S) were included. Of them, 14 were AMC, 7 were SMC and 4 CTR. Demographic and clinical data are shown in Table 1. We observed group differences in age (Kruskal-Wallis test $\mathrm{p}<0.05$ ). CTR and AMC differed in EYO (Mann-Whitney $\mathrm{U}, \mathrm{p}<0.01$ ), but not in age. In our sample, we observed a bias towards female participants due to the fact that women from our ADAD clinical cohort were more prone to participate in research studies than men. Seven AMC were classified as stage $I-A M C$, younger than 15 years from estimated onset (mean $\mathrm{EYO}=-18.8$ years, SD: 3.16), and $7 \mathrm{AMC}$ were classified as stage $I I-A M C$, older than 15 years from estimated onset (mean $\mathrm{EYO}=-7.95$ years, SD: 7.14).

\subsection{Safety results.}

No AE were reported related to the tracer injection or PET scanning. 


\subsection{Visual assessment of PET images.}

Sixteen mutation carriers, all $7 \mathrm{SMC}$ and 9 out of $14 \mathrm{AMC}$, showed abnormal (increased)

FBB uptake at visual assessment at least in one of the areas studied. Seven of them (43.75\%) showed both generalized uniform uptake and local striatal tracer uptake. They corresponded to 4 SMC and 3 AMC with EYO range -13 to 9.4 years and the following mutations: S169P, L173F, E120K, H163R, K239N, L173F. Two L282R AMC (12.5\%) presented only generalized uniform cortical uptake. Two SMC (12.5\%) with EYO 3.1 and 4 years and G209E and H163R mutations showed only striatal retention. Four AMC (25\%) had focal cortical retention, with EYO range to -20.2 to -1.8 years and the K239N, L282R, G378R and V151M mutations. One SMC (6.25\%) with EYO 7.3 and L286P mutation presented cerebellar tracer retention in addition to generalized uniform cortical uptake. This participant was the only SMC who did not show striatal uptake at visual inspection. The 9 participants with high striatal uptake were 6 SMC and 3 AMC. The 5 remaining AMC (EYO range: -24.4 to -15.7) were negative in all the areas evaluated at visual inspection. Figure 1 provides one example of each of the amyloid patterns observed. All non-carriers were negative at visual inspection.

\subsection{Individual quantification}

We studied the individual FBB uptake quantifications in all the regions. The percentages of subjects with altered (above cutoff) values within each group and for each region are summarized in Figure 2. All SMC subjects showed regional SUVR values above the cutoff in all the areas studied except for caudate ( $85.7 \%$ positive subjects), putamen ( $85.7 \%$ positive), hippocampus ( $14.2 \%$ positive), amygdala ( $57.4 \%$ positive), enotorhinal cortex ( $0 \%$ positive), parahippocampal cortex $(57.14 \%$ positive $)$ and temporal pole $(71.43 \%$ positive $)$. Interestingly, the only SMC (EYO= 7.32 years, mutation $\mathrm{L} 268 \mathrm{P}$, and $\mathrm{CDR}=1)$ subject that did not show increased SUVR in caudate showed increased SUVR in the cerebellum. The youngest AMC who showed FBB above cutoff had an EYO of -20 years ( 7 out of 40 positive regions), and the 2 nd youngest had an EYO of -16 years (20 out of 40 positive regions). 
Of the stage I-AMC subjects, only the two subjects mentioned above, showed SUVR above cutoff in some areas: bankssts, precuneus, inferior and superior parietal, supramarginal and lateral occipital (Figure 2). However, all the stage II-AMC subjects presented at least one area with SURV above the cutoff. CTR subjects present normal SURV values in all the areas except for the pallidum.

The results obtained using the original methods defined in Bullich et al. (2017) are included in Supplementary Material, together with the comparative analysis between both sets of results. In summary, we found a strong correlation between both sets of measures, and we obtained similar results both for group comparison analyses and in the rate of positive/negative subjects per region. We also tested the regional variability of the thresholds and we concluded that the number of subjects classified as positive or negative was almost identical when using regional vs global thresholds (See Supplementary Material for results and further discussion).

\subsection{Group differences in SUVR.}

In SMC, we found higher FBB uptake in all the regions, compared with $\underline{\mathrm{CTR} \text { (all surviving }}$ FDR threshold: $p=0.023$, Figure 3). In AMC, the caudate and all cortical areas, with the exception of the entorhinal cortex, showed increased SUVR compared with CTR (all surviving FDR threshold: $p=0.019$, Figure 3). When AMC subjects were divided according to EYO, we found increased uptake ( $\mathrm{p}<0.05$, uncorrected) in stage I-AMC compared to CTR in 21 out of 40 regions including 20 cortical ROIS and the caudate. However, none of these differences survived FDR correction. In stage II-AMC, compared with CTR, we also found higher uptake in the hippocampus and in the rest of cortical areas (all surviving FDR threshold: $p=0.037$, Figure $4 \mathrm{~A}$ and supplementary material).

\subsection{Correlation between SUVR and EYO.}

For all MC, we observed significant correlations between SUVR and EYO in all the areas studied (all surviving FDR threshold: $p=0.0023$, see values in supplementary material). In 
AMC, we found correlations between SUVR and EYO in many cortical areas and in the amygdala (all surviving FDR threshold: $\mathrm{p}=0.035$ ), which were not seen in SMC. The regions with the strongest significant correlations $(r>0.62, p<0.05$, FDR corrected) are shown in Figure 5.

\subsection{Cortical Thickness}

In AMC, we found higher CTh compared to CTR in bankssts, paracentral, parsopercularis, parstriangularis, rostral middle frontal, and superior temporal ( $\mathrm{p}<0.05$, Figure 4B). In SMC, we found thinner cortex compared to controls in precuneus and inferior parietal, superior parietal and supramarginal regions (all $\mathrm{p}<0.05$, Figure 4B). We found negative correlation with $\mathrm{EYO}$ in MC in the bankssts, the fusiform, the inferior parietal, the middle temporal, the postcentral, the precuneus and the supramarginal. We observed that all the areas mentioned above, with significantly increased CTh in AMC or decreased CTh in SMC, showed also significant A $\beta$ deposition compared with CTR (Figure 4).

\section{DISCUSSION}

We examined the spatial pattern of FBB retention in ADAD due to PSEN1 mutations. Consistent with the amyloid hypothesis and according with previous findings with other tracers and in other cohorts, we found increased FBB uptake in PSEN1 mutation carriers in cortical and subcortical areas, found in precuneus, bankssts, supramarginal, superior and inferior parietal and lateral occipital in asymptomatic subjects younger than two decades before the predicted symptoms onset, suggesting progressive deposition of fibrillar $A \beta$. We identified areas with strong correlation between FBB uptake and EYO among which outstands the rostral anterior cingulate, suggesting that this area could be used to track the progressive fibrillar $\mathrm{A} \beta$ deposition across the disease. Striatal pathological uptake was observed in $50 \%$ of the positive scans and cerebellar uptake was only in one SMC at visual examination. In addition, we identified areas of increased CTh in AMC, in accordance with 
previous studies (Fortea et al., 2010; Sala-Llonch et al., 2015), as the bankssts and the precuneus, which also presented significant amyloid deposition in early AMC stages. Besides not being a longitudinal study, our findings in subjects at different EYO suggest a progressive increase in amyloid deposition when approaching disease onset. In terms of safety, the use of FBB PET was shown to be safe and well-tolerated in this population.

Overall, we demonstrate the capability of FBB-PET imaging to depict amyloid deposition changes up to 20 years before the clinical onset of AD. Notably, we described regional patterns similar than those reported in other cohorts with other tracers, besides potential differences in the degree of retention between amyloid binding detected with 18C-PIB and FBB (Villemagne et al., 2012). Studies on the DIAN cohort, both cross-sectionally (Bateman et al., 2012; Benzinger et al., 2013; Oxtoby et al., 2018), and longitudinally (Gordon et al., 2018) reported amyloid deposition in nearly every cortical region 15 years before the estimated age of onset for ADAD mutation carriers, measured with PIB-PET. Also using PIB, another study reported higher striatum uptake in presymptomatic ADAD subjects 17 years before the estimated onset and longitudinal increases in frontal cortex and in putamen when subjects approached the age of onset (Rodriguez-Vieitez et al., 2016). In presymptomatic PSEN1 E280A mutation carriers, using 18F-florbetapir, it has been found that fibrillar A $\beta$ began to accumulate about 16 years before the predicted median age of mild cognitive impairment onset, in anterior and posterior cingulate, precuneus, and parietotemporal and frontal cortex, and in basal ganglia (Fleisher et al., 2015). In addition, studies in sporadic AD (Villemagne et al., 2013) have found amyloid PET changes up to 17 years before the stage of mild dementia, suggesting similarities between $\mathrm{ADAD}$ and late-onset sporadic $\mathrm{AD}$ in the chronology of the progressive amyloid deposition.

As regards the regional specificity, using PIB, striatal amyloid accumulation was described as a specific feature of ADAD (Klunk et al., 2007). In our sample, using FBB, we find a regional pattern of alterations in $\mathrm{ADAD}$, which was in general characterized by diffuse cortical uptake 
accompanied by slight striatal alterations. Striatal uptake was frequent but not universal in our subjects. Interestingly, the only SMC who did not show altered FBB uptake in the striatum showed relevant cerebellar uptake. Increased FBB uptake in caudate appeared in the early presymptomatic phase, while hippocampal uptake increase appeared in the last years of the presymptomatic stage, it was significantly higher in stage II-AMC (less than 15 years before the predicted age of onset), with no differences in younger asymptomatic carriers, compared to non-carriers. Additionally, only one of the mutation carriers showed FBB uptake above the cut-off in the hippocampus. Our study did not show relevant amyloid tracer retention in ADAD in the hippocampus, similar to previous studies (Benzinger et al., 2013; Gordon et al., 2018; Rodríguez-Vietez et al., 2016) and in agreement to classical neuropathological studies that revealed the hippocampus only presented mild deposits of amyloid (Braak and Braak, 1991). The rest of subcortical structures studied showed abnormal deposition in SMC, but not in AMC.

We found strong correlations between FBB uptake and $\mathrm{EYO}$ for all $\mathrm{MC}$ in all the regions studied. These were also observed in AMC, but not in SMC, suggesting that time does not relevantly affect amyloid accumulation after the symptoms onset. If we exclude the pallidum, which may present unspecific binging, considering that CTR also presented high uptake, the anterior rostral cingulate appeared as the region showing strongest correlations in AMC, and thus it can be considered as a candidate region to be used to track amyloid deposition (rho $=$ 0.75 in $\mathrm{AMC}$ and 0.83 in $\mathrm{MC}$ ). In addition, the precuneus that has been proposed as a candidate region by other authors (Bateman et al., 2012; Benzinger et al., 2013; Gordon et al., 2018), also showed strong correlations in our sample (rho $=0.62$ in $\mathrm{AMC}$ and 0.77 in $\mathrm{MC}$ ). In previous studies, we described increased CTh in AMC compared with controls, at a mean of 16 years from symptoms onset, in temporoparietal cortex, precentral and postcentral cortices, and pars triangularis and pars opercularis regions, accompanied by increased caudate volume (Fortea et al., 2010; Sala-Llonch et al., 2015). We then speculated that neuroinflammation and/or accumulation of amyloid species preceding neurodegeneration could account for this phenomenon. Here, we observed that those regions showed increased 
FBB uptake very early on in the disease course, suggesting pathological fibrillar amyloid deposition in areas with increased thickness (Fortea et al., 2010; Sala-Llonch et al., 2015). In the same line, Rodriguez-Vieitez and collaborators (Rodriguez-Vieitez et al., 2016) described that initially high followed by declining astrocytosis in $\mathrm{ADAD}$ carriers, at the time of early amyloid deposition, suggesting astrocyte activation is implicated in the early stages of AD pathology (Quiroz et al., 2018).

Notably, in this study, we used a semi-automated method that uses individual anatomical information, PET-MRI registration, and PVE correction (Greve et al., 2016). Even if we have proven that the classification into positive/negative scans is similar to that obtained by visual ratings, automatic quantifications allowed us providing an accurate description of regional alterations, discriminating areas affected differently at each stage of the disease progression.

It is a matter of discussion whether different amyloid tracers bind to identical sites on $\mathrm{A} \beta$ fibrils, offering the same ability to detect the regional $A \beta$ burden or not. In this sense, one of the main contributions of our work is the confirmation that FBB is able to detect the amyloid plaques observed in PSEN1 carriers as soon as two decades before predicted age of onset. On the other hand, previous studies refer to the precuneus as the preferred area to study the progressive deposition of amyloid using amyloid PET. Here, besides validating the results on the precuneus, we discuss that other areas, as the anterior cingulate, are also good candidates to monitor amyloid uptake.

Overall, even when most of our results are in line with previous studies with other tracers, suggesting that most of the effects described are related to real amyloid deposition and not to an unspecific tracer effect, we should acknowledge some limitations. First, the sample size limits the strength of the results and precluded some of the potential analysis, but on the other hand, it is relevant considering that is a unicentric study and no other data are available with FBB in this population. The second limitation is that this is a cross-sectional study and even if 
cross-sectional studies have provided evidences further proved in longitudinal studies, the magnitude of the effect or the temporal pattern of changes may differ with real longitudinal studies. Other limitation is the use of a FBB cut-off positivity that was obtained for sporadic AD. However, the results obtained are congruent with what could be expected according to the literature. Finally, our approach relied upon a familial age of symptom onset as a proxy for disease progression, which limits the accuracy of predictions due to the potential imprecision in such estimates (Ryman et al., 2014). Giving that it is currently not possible to predict the exact age of onset for each AMC subject using individual biomarkers, in the future our results would need to be re-analyzed retrospectively when these AMC reach the disease onset.

\section{Conclusion}

In summary, we confirm and expand previous findings of very early, progressive and region dependent amyloid tracer uptake in ADAD in an independent unicentric cohort (Barcelona cohort), with a different amyloid tracer, FBB. Our findings would suggest that FBB disclose a similar spatial pattern and chronology of tracer uptake to that described in previous publications with other amyloid tracers in ADAD.

\section{Acknowledgments}

We thank all the volunteers for their participation in this study.

\section{Disclosure statement}

This work was supported by the Instituto Carlos III (PI12/00013 and PI16/00235 to RSV, cofunded by the European Regional Development Fund), by the Departament de Salut de la Generalitat de Catalunya (PERIS 2016-2020 SLT002/16/00329, to AA) and by an Investigator Sponsored Study founded by Life Molecular Imaging. 
Life Molecular Imaging provided the FBB doses free of charge and funded the costs required by the Spanish Regulatory Agency (Investigator Sponsored Study (ISS) protocol code FBBFAD-2014; EudraCT No. 2014-000763-41). The authors declare no competing interests. 


\section{REFERENCES}

Bateman, R.J., Aisen, P.S., De Strooper, B., Fox, N.C., Lemere, C.A., Ringman, J.M., Salloway, S., Sperling, R.A., Windisch, M., Xiong, C., 2011. Autosomal-dominant Alzheimer's disease: a review and proposal for the prevention of Alzheimer's disease. Alzheimers. Res. Ther. 3, 1. doi:10.1186/alzrt59

Bateman, R.J., Xiong, C., Benzinger, T.L.S., Fagan, A.M., Goate, A., Fox, N.C., Marcus, D.S., Cairns, N.J., Xie, X., Blazey, T.M., Holtzman, D.M., Santacruz, A., Buckles, V., Oliver, A., Moulder, K., Aisen, P.S., Ghetti, B., Klunk, W.E., McDade, E., Martins, R.N., Masters, C.L., Mayeux, R., Ringman, J.M., Rossor, M.N., Schofield, P.R., Sperling, R.A., Salloway, S., Morris, J.C., 2012. Clinical and Biomarker Changes in Dominantly Inherited Alzheimer's Disease. N. Engl. J. Med. 367, 795-804. doi:10.1056/NEJMoa1202753

Benjamini, Y., Hochberg, Y., 1995. Controlling the false discovery rate: A practical and powerful approach to multiple testing. Journal of the Royal Statistical Society, Series B (Methodological). 57(1), 289-300.

Braak H., Braak E., 1991. Neuropathological stageing of Alzheimer-related changes. Acta Neuropathol.82(4):239-59.

Benzinger, T.L.S., Blazey, T., Jack, C.R., Koeppe, R.A., Su, Y., Xiong, C., Raichle, M.E., Snyder, A.Z., Ances, B.M., Bateman, R.J., Cairns, N.J., Fagan, A.M., Goate, A., Marcus, D.S., Aisen, P.S., Christensen, J.J., Ercole, L., Hornbeck, R.C., Farrar, A.M., Aldea, P., Jasielec, M.S., Owen, C.J., Xie, X., Mayeux, R., Brickman, A., McDade, E., Klunk, W., Mathis, C.A., Ringman, J., Thompson, P.M., Ghetti, B., Saykin, A.J., Sperling, R.A., Johnson, K.A., Salloway, S., Correia, S., Schofield, P.R., Masters, C.L., Rowe, C., Villemagne, V.L., Martins, R., Ourselin, S., Rossor, M.N., Fox, N.C., Cash, D.M., Weiner, M.W., Holtzman, D.M., Buckles, V.D., Moulder, K., Morris, J.C., 2013. Regional variability of imaging biomarkers in autosomal dominant Alzheimer's disease. Proc. Natl. Acad. Sci. U. S. A. 110, E4502-9. doi:10.1073/pnas.1317918110

Bullich, S., Seibyl, J., Catafau, A.M., Jovalekic, A., Koglin, N., Barthel, H., Sabri, O., De Santi, S., 2017. Optimized classification of 18 F-Florbetaben PET scans as positive and negative using an SUVR quantitative approach and comparison to visual assessment. NeuroImage Clin. 15, 325-332. doi:10.1016/j.nicl.2017.04.025

Carrillo, M.C., Brashear, H.R., Logovinsky, V., Ryan, J.M., Feldman, H.H., Siemers, E.R., Abushakra, S., Hartley, D.M., Petersen, R.C., Khachaturian, A.S., Sperling, R.A., 2013. Can we prevent Alzheimer's disease? Secondary “prevention" trials in Alzheimer's disease. Alzheimer's Dement. 9, 123-131.e1. doi:10.1016/j.jalz.2012.12.004 
Fischl, B., Salat, D.H., Busa, E., Albert, M., Dieterich, M., Haselgrove, C., van der Kouwe, A., Killiany, R., Kennedy, D., Klaveness, S., Montillo, A., Makris, N., Rosen, B., Dale, A.M., 2002. Whole brain segmentation: automated labeling of neuroanatomical structures in the human brain. Neuron 33, 341-55.

Fischl, B., Salat, D.H., van der Kouwe, A.J.W., Makris, N., Ségonne, F., Quinn, B.T., Dale, A.M., 2004. Sequence-independent segmentation of magnetic resonance images. Neuroimage 23 Suppl 1, S69-84. doi:10.1016/j.neuroimage.2004.07.016 Fleisher, A.S., Chen, K., Quiroz, Y.T., Jakimovich, L.J., Gomez, M.G., Langois, C.M., Langbaum, J.B., Ayutyanont, N., Roontiva, A., Thiyyagura, P., Lee, W., Mo, H., Lopez, L., Moreno, S., Acosta-Baena, N., Giraldo, M., Garcia, G., Reiman, R.A., Huentelman, M.J., Kosik, K.S., Tariot, P.N., Lopera, F., Reiman, E.M., 2012. Florbetapir PET analysis of amyloid- $\beta$ deposition in the presenilin 1 E280A autosomal dominant Alzheimer's disease kindred: a cross-sectional study. Lancet Neurol. 11, 1057-1065. doi:10.1016/S1474-4422(12)70227-2

Fleisher, A.S., Chen, K., Quiroz, Y.T., Jakimovich, L.J., Gutierrez Gomez, M., Langois, C.M., Langbaum, J.B.S., Roontiva, A., Thiyyagura, P., Lee, W., Ayutyanont, N., Lopez, L., Moreno, S., Muñoz, C., Tirado, V., Acosta-Baena, N., Fagan, A.M., Giraldo, M., Garcia, G., Huentelman, M.J., Tariot, P.N., Lopera, F., Reiman, E.M., 2015.

Associations between biomarkers and age in the presenilin 1 E280A autosomal dominant Alzheimer disease kindred: A cross-sectional study. JAMA Neurol. 72, 316324. doi:10.1001/jamaneurol.2014.3314

Fortea, J., Lladó, A., Clarimón, J., Lleó, A., Oliva, R., Peri, J., Pintor, L., Yagüe, J., Blesa, R., Molinuevo, J.L., Sánchez-Valle, R., 2011. PICOGEN: five years experience with a genetic counselling program for dementia. Neurología 26, 143-9. doi:10.1016/j.nrl.2010.09.011

Fortea, J., Sala-Llonch, R., Bartrés-Faz, D., Bosch, B., Lladó, A., Bargalló, N., Molinuevo, J.L., Sánchez-Valle, R., 2010. Increased cortical thickness and caudate volume precede atrophy in PSEN1 mutation carriers. J. Alzheimers. Dis. 22, 909-22. doi:10.3233/JAD2010-100678

Gordon, B.A., Blazey, T.M., Su, Y., Hari-Raj, A., Dincer, A., Flores, S., Christensen, J., McDade, E., Wang, G., Xiong, C., Cairns, N.J., Hassenstab, J., Marcus, D.S., Fagan, A.M., Jack, C.R., Hornbeck, R.C., Paumier, K.L., Ances, B.M., Berman, S.B., Brickman, A.M., Cash, D.M., Chhatwal, J.P., Correia, S., Förster, S., Fox, N.C., GraffRadford, N.R., la Fougère, C., Levin, J., Masters, C.L., Rossor, M.N., Salloway, S., Saykin, A.J., Schofield, P.R., Thompson, P.M., Weiner, M.M., Holtzman, D.M., Raichle, M.E., Morris, J.C., Bateman, R.J., Benzinger, T.L.S., 2018. Spatial patterns of neuroimaging biomarker change in individuals from families with autosomal dominant 
Alzheimer's disease: a longitudinal study. Lancet Neurol. 17, 241-250.

doi:10.1016/S1474-4422(18)30028-0

Greve, D.N., Fischl, B., 2009. Accurate and robust brain image alignment using boundarybased registration. Neuroimage 48, 63-72. doi:10.1016/j.neuroimage.2009.06.060

Greve, D.N., Salat, D.H., Bowen, S.L., Izquierdo-Garcia, D., Schultz, A.P., Catana, C., Becker, J.A., Svarer, C., Knudsen, G.M., Sperling, R.A., Johnson, K.A., 2016. Different partial volume correction methods lead to different conclusions: An 18 F-FDG-PET study of aging. Neuroimage 132, 334-343. doi:10.1016/j.neuroimage.2016.02.042

Klunk, W.E., Price, J.C., Mathis, C.A., Tsopelas, N.D., Lopresti, B.J., Ziolko, S.K., Bi, W., Hoge, J.A., Cohen, A.D., Ikonomovic, M.D., Saxton, J.A., Snitz, B.E., Pollen, D.A., Moonis, M., Lippa, C.F., Swearer, J.M., Johnson, K.A., Rentz, D.M., Fischman, A.J., Aizenstein, H.J., DeKosky, S.T., 2007. Amyloid Deposition Begins in the Striatum of Presenilin-1 Mutation Carriers from Two Unrelated Pedigrees. J. Neurosci. 27, 61746184. doi:10.1523/JNEUROSCI.0730-07.2007

Mills, S.M., Mallmann, J., Santacruz, A.M., Fuqua, A., Carril, M., Aisen, P.S., Althage, M.C., Belyew, S., Benzinger, T.L., Brooks, W.S., Buckles, V.D., Cairns, N.J., Clifford, D., Danek, A., Fagan, A.M., Farlow, M., Fox, N., Ghetti, B., Goate, A.M., Heinrichs, D., Hornbeck, R., Jack, C., Jucker, M., Klunk, W.E., Marcus, D.S., Martins, R.N., Masters, C.M., Mayeux, R., McDade, E., Morris, J.C., Oliver, A., Ringman, J.M., Rossor, M.N., Salloway, S., Schofield, P.R., Snider, J., Snyder, P., Sperling, R.A., Stewart, C., Thomas, R.G., Xiong, C., Bateman, R.J., 2013. Preclinical trials in autosomal dominant AD: implementation of the DIAN-TU trial. Rev. Neurol. (Paris). 169, 737-43. doi:10.1016/j.neurol.2013.07.017

Oxtoby, N.P., Young, A.L., Cash, D.M., Benzinger, T.L.S., Fagan, A.M., Morris, J.C., Bateman, R.J., Fox, N.C., Schott, J.M., Alexander, D.C., 2018. Data-driven models of dominantly-inherited Alzheimer's disease progression. Brain 141, 1529-1544. doi:10.1093/brain/awy050

Quiroz, Y.T., Sperling, R.A., Norton, D.J., Baena, A., Arboleda-Velasquez, J.F., Cosio, D., Schultz, A., Lapoint, M., Guzman-Velez, E., Miller, J.B., Kim, L.A., Chen, K., Tariot, P.N., Lopera, F., Reiman, E.M., Johnson, K.A., 2018. Association between amyloid and tau accumulation in young adults with autosomal dominant Alzheimer disease. JAMA Neurol. 75, 548-556. doi:10.1001/jamaneurol.2017.4907

Rodriguez-Vieitez, E., Saint-Aubert, L., Carter, S.F., Almkvist, O., Farid, K., Schöll, M., Chiotis, K., Thordardottir, S., Graff, C., Wall, A., Långström, B., Nordberg, A., 2016. Diverging longitudinal changes in astrocytosis and amyloid PET in autosomal dominant Alzheimer's disease. Brain 139, 922-936. doi:10.1093/brain/awv404 
Rowe, C.C., Doré, V., Jones, G., Baxendale, D., Mulligan, R.S., Bullich, S., Stephens, A.W., De Santi, S., Masters, C.L., Dinkelborg, L., Villemagne, V.L., 2017. 18F-Florbetaben PET beta-amyloid binding expressed in Centiloids. Eur. J. Nucl. Med. Mol. Imaging 44, 2053-2059. doi:10.1007/s00259-017-3749-6

Ryman, D.C., Acosta-Baena, N., Aisen, P.S., Bird, T., Danek, A., Fox, N.C., Goate, A., Frommelt, P., Ghetti, B., Langbaum, J.B.S., Lopera, F., Martins, R., Masters, C.L., Mayeux, R.P., McDade, E., Moreno, S., Reiman, E.M., Ringman, J.M., Salloway, S., Schofield, P.R., Sperling, R., Tariot, P.N., Xiong, C., Morris, J.C., Bateman, R.J., Dominantly Inherited Alzheimer Network, 2014. Symptom onset in autosomal dominant Alzheimer disease: a systematic review and meta-analysis. Neurology 83, 253-60. doi:10.1212/WNL.0000000000000596

Sala-Llonch, R., Lladó, A., Fortea, J., Bosch, B., Antonell, A., Balasa, M., Bargalló, N., Bartrés-Faz, D., Molinuevo, J.L., Sánchez-Valle, R., 2015. Evolving brain structural changes in PSEN1 mutation carriers. Neurobiol. Aging 36, 1261-1270.

doi:10.1016/j.neurobiolaging.2014.12.022

Su, Y., Blazey, T.M., Owen, C.J., Christensen, J.J., Friedrichsen, K., Joseph-Mathurin, N., Wang, Q., Hornbeck, R.C., Ances, B.M., Snyder, A.Z., Cash, L.A., Koeppe, R.A., Klunk, W.E., Galasko, D., Brickman, A.M., McDade, E., Ringman, J.M., Thompson, P.M., Saykin, A.J., Ghetti, B., Sperling, R.A., Johnson, K.A., Salloway, S.P., Schofield, P.R., Masters, C.L., Villemagne, V.L., Fox, N.C., Förster, S., Chen, K., Reiman, E.M., Xiong, C., Marcus, D.S., Weiner, M.W., Morris, J.C., Bateman, R.J., Benzinger, T.L.S., Dominantly Inherited Alzheimer Network, 2016. Quantitative Amyloid Imaging in Autosomal Dominant Alzheimer's Disease: Results from the DIAN Study Group. PLoS One 11, e0152082. doi:10.1371/journal.pone.0152082

Tzourio-Mazoyer, N., Landeau, B., Papathanassiou, D., Crivello, F., Etard, O., Delcroix, N., Mazoyer, B., Joliot, M, 2002. Automated anatomical labeling of activations in SPM using a macroscopic anatomical parcellation of the MNI MRI single-subject brain. Neuroimage. 15(1):273-89.

Villemagne, V.L., Burnham, S., Bourgeat, P., Brown, B., Ellis, K.A., Salvado, O., Szoeke, C., Macaulay, S.L., Martins, R., Maruff, P., Ames, D., Rowe, C.C., Masters, C.L., 2013. Amyloid $\beta$ deposition, neurodegeneration, and cognitive decline in sporadic Alzheimer's disease: a prospective cohort study. Lancet Neurol. 12, 357-67. doi:10.1016/S1474-4422(13)70044-9

Villemagne, V.L., Mulligan, R.S., Pejoska, S., Ong, K., Jones, G., O’Keefe, G., Chan, J.G., Young, K., Tochon-Danguy, H., Masters, C.L., Rowe, C.C., 2012. Comparison of 11C$\mathrm{PiB}$ and $18 \mathrm{~F}$-florbetaben for $\mathrm{A} \beta$ imaging in ageing and Alzheimer's disease. Eur. J. Nucl. Med. Mol. Imaging 39, 983-989. doi:10.1007/s00259-012-2088-x 


\section{TABLES :}

\begin{tabular}{|l|c|c|c|c|}
\hline & CTR & AMC & SMC & P \\
\hline $\mathrm{N}=25$ & 4 & 14 & 7 & \\
\hline Age, y, mean (SD) & $48.37(10.01)$ & $37.11(8.79)$ & $51.88(2.32)$ & $\mathrm{p}=0.005$ \\
\hline Sex, M/F & $1 / 3$ & $1 / 13$ & $5 / 2$ & $\mathrm{p}=0.01$ \\
\hline EYO, y, mean (SD) & $2.06(4.56)$ & $-13.38(7.73)^{*}$ & $4.88(2.58)$ & $\mathrm{p}<0.001$ \\
\hline MMSE, mean (SD) & 30 & $28.86(1.56)$ & $22.43(3.31)$ & $\mathrm{p}<0.001$ \\
\hline CDR total, mean (SD) & 0 & 0 & $1.07(0.67)$ & $\mathrm{p}<0.001$ \\
\hline CDR-SOB, mean (SD) & 0 & 0 & $4.57(3.62)$ & $\mathrm{p}<0.001$ \\
\hline
\end{tabular}

Table 1. Participant demographics and clinical variables.

CTR: non-carriers; AMC: asymptomatic mutation carriers; SMC: symptomatic mutation carriers; SD: standard deviation, P values obtained by Kruskal-Wallis test. * indicates that there are differences between AMC and CTR 


\section{FIGURE LEGENDS:}

Figure 1. Visual patterns of FBB uptake observed in the PSEN1 mutation carriers. A). Negative scan. B). Generalized uniform uptake C). Focal occipital uptake. D). Striatal and focal cortical uptake. E). Cerebellar uptake.

Figure 2. Percentage of subjects with increase individual SUVR values $($ cutoff $=0.78)$ for each region at each stage. On the right, representation of the percentages on a standard surface brain.

Figure 3. Mean SUVR within each ROI for controls (CTR), asymptomatic mutation carriers (AMC) and symptomatic mutation carriers (SMC). Only areas with significant differences between CTR and AMC are shown in the figure. Differences between CTR and SMC are significant for all regions studied.

Figure 4. Summary of FBB and CTh group comparisons. In (A), areas with significant FBB uptake differences in the comparison with the CTR group $(\mathrm{p}<0.05$ in the Kruskal-Wallis test), as indicated with gray boxes (light gray for stage I AMC, medium gray for stage II AMC and dark gray for SMC). The brain representation shows significant cortical parcellations on a standard surface brain. Green-yellow scale indicates the degree of differences in SUVR for each region of each group compared to CTR. In (B), areas with significant CTh differences in the comparison with the CTR group ( $<<0.05$ in the Kruskal-Wallis test), as indicated with blue boxes (decreased $\mathrm{CTh}$ ) and red boxes (increased $\mathrm{CTh}$ ). The brain representation shows significant cortical parcellations on a standard surface brain. Blue-Red colors indicate the direction of differences for each region of each group compared to CTR, following the same legend as the box grid. See main text for differences surviving multiple comparisons.

Figure 5. Correlation between SUVR and EYO for each group. For each region, the result of the Spearman correlation within the AMC group is shown on the bottom-right corner. Only regions with correlation of $r>0.62$ are shown and corrected $p<0.05$. The detailed results of the correlations for all groups and all regions are shown in Table 2. 


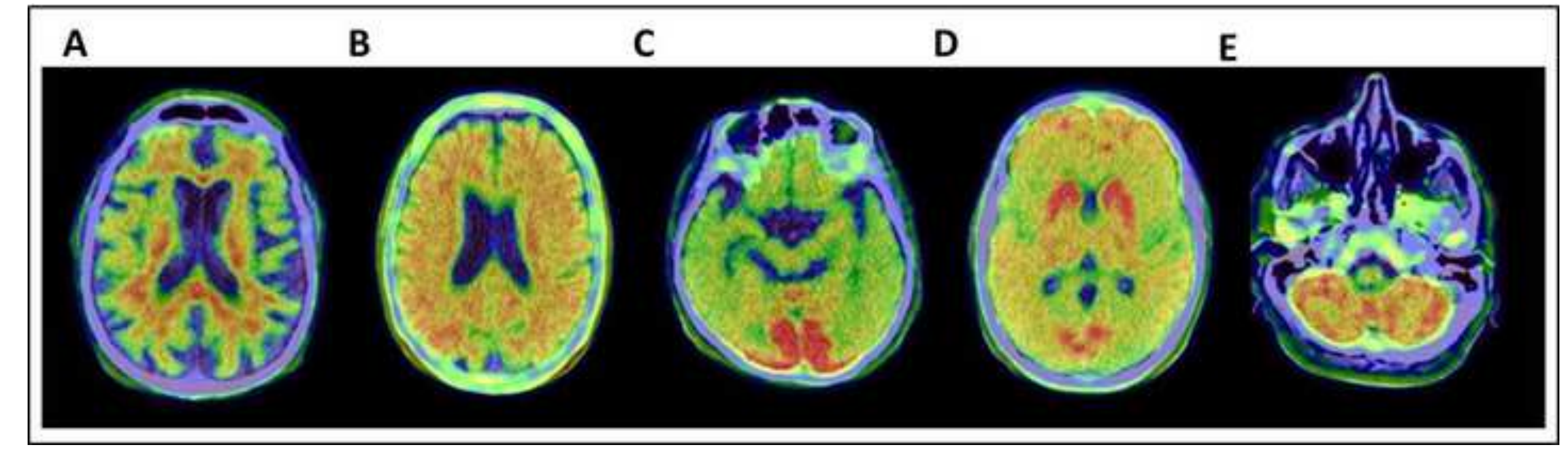

\section{Click here to download high resolution image}




\section{gure 2}

Click here to download high resolution image

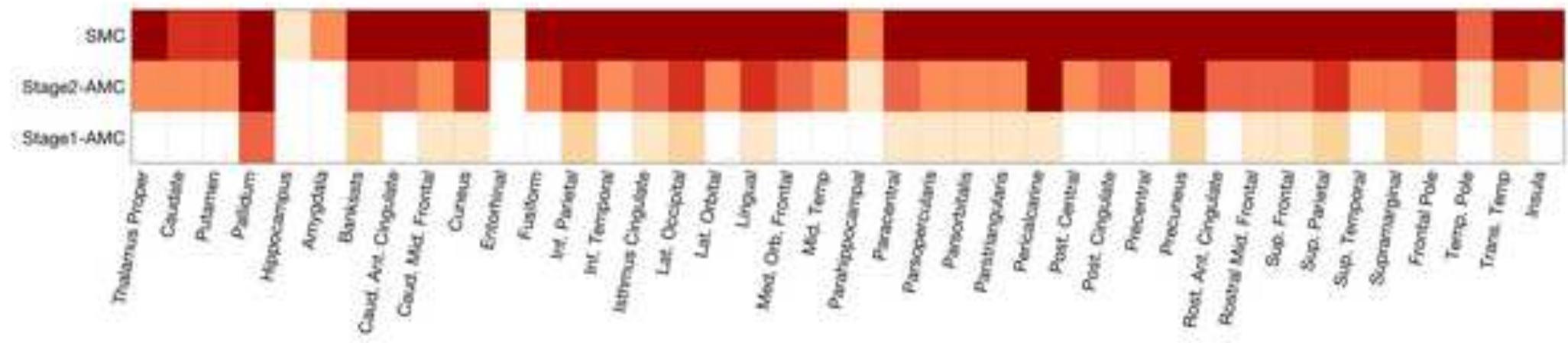

Whastere $30 \quad 100$ 


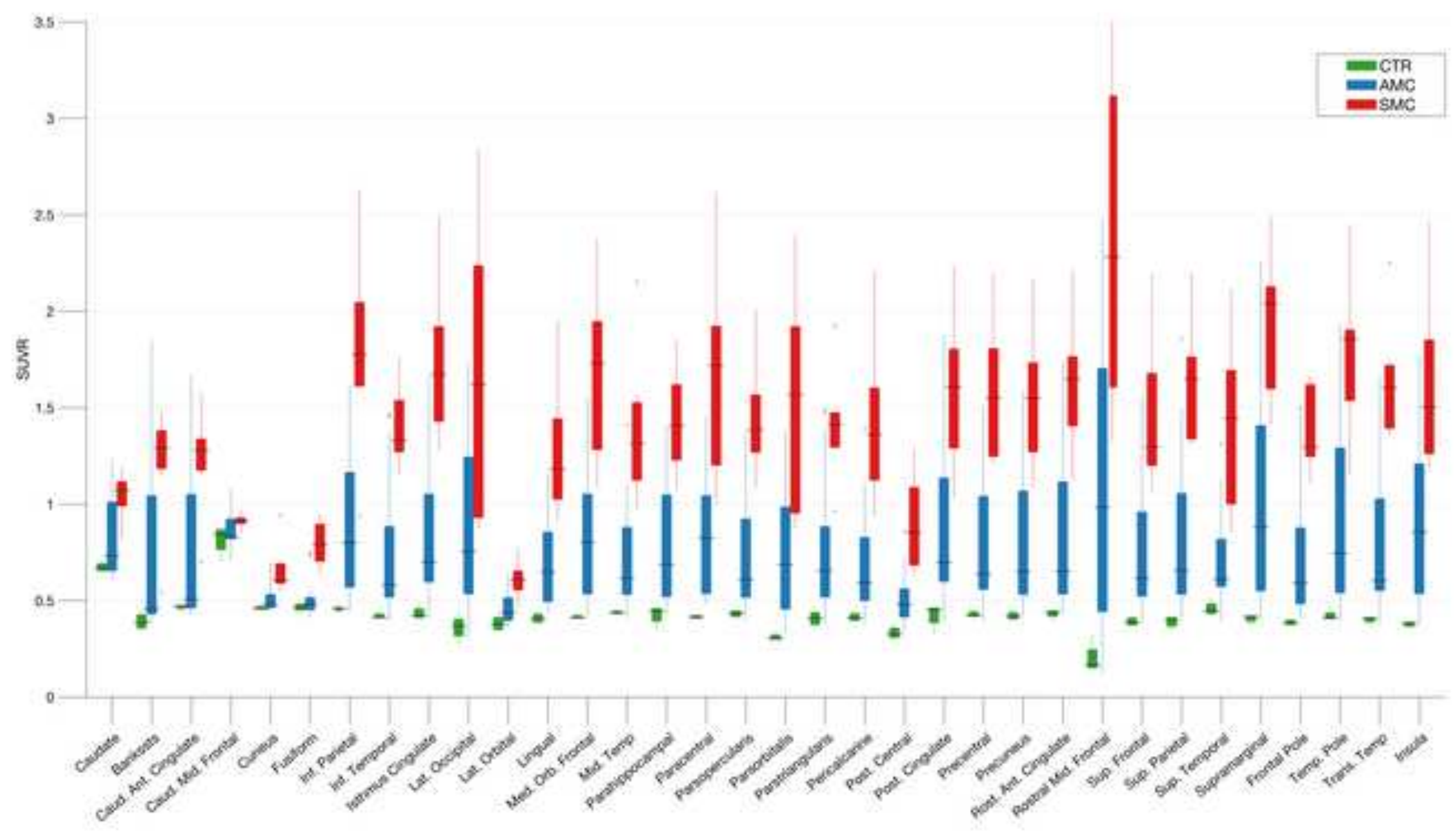


Click here to download high resolution image

\section{A. FBB-PET Results}

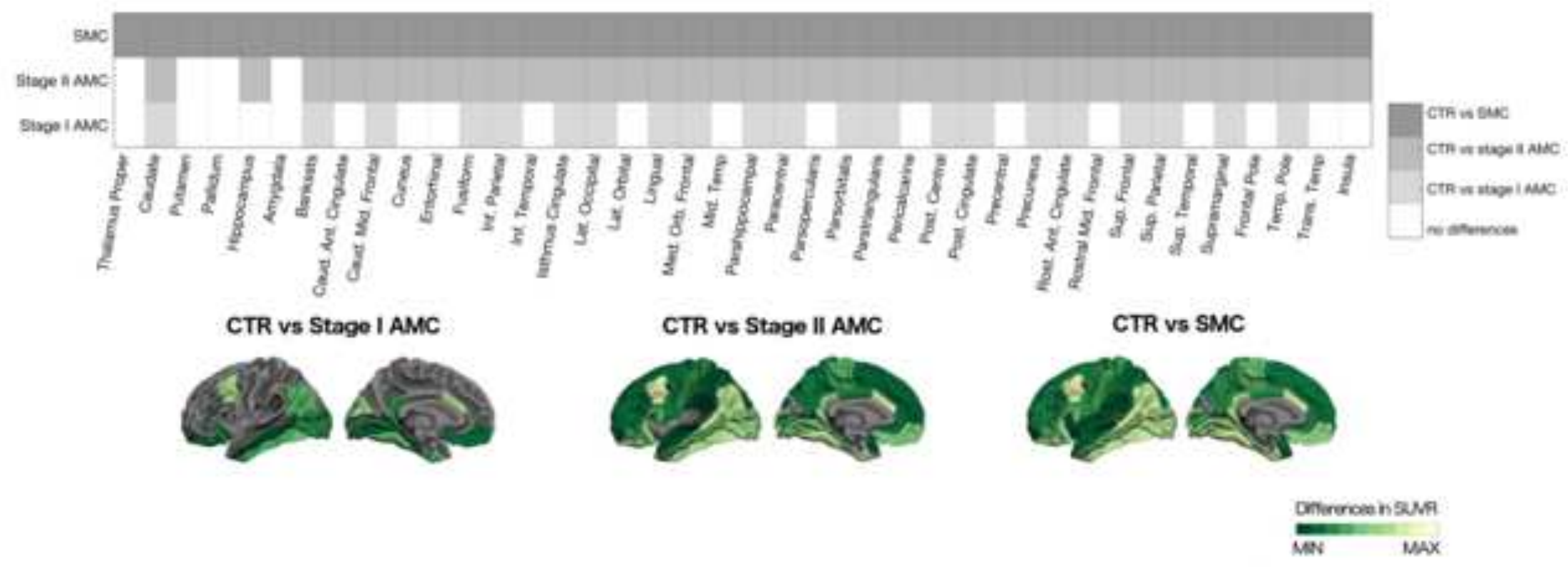

\section{B. Cortical Thickness Results}

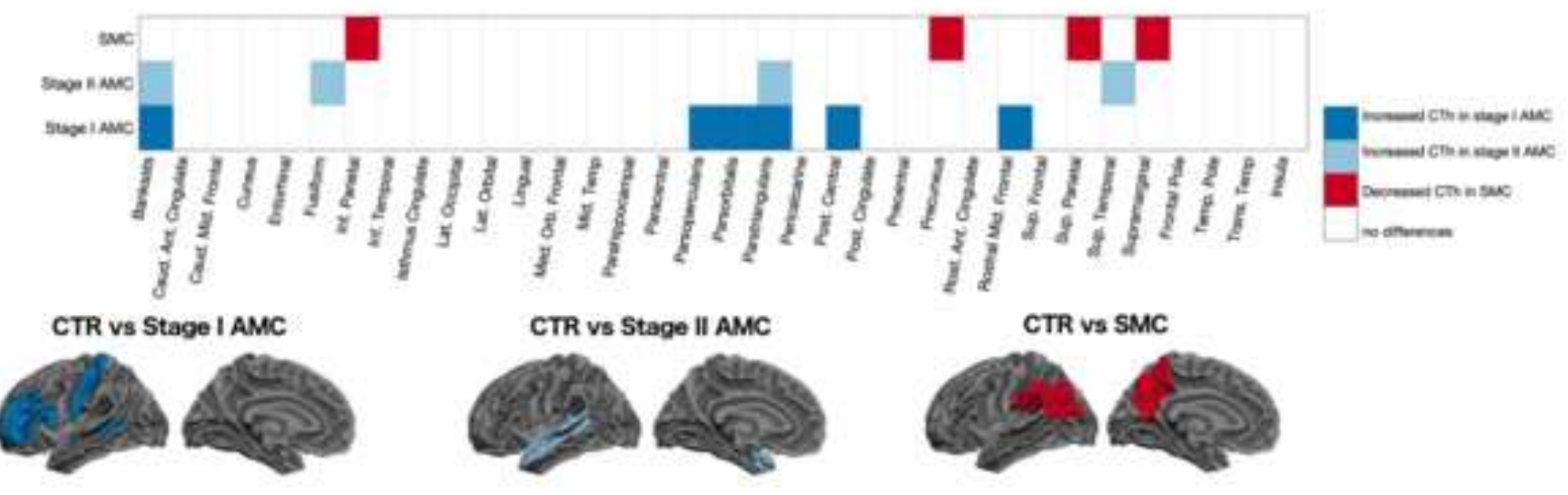



Supplemental or Multimedia Files
Click here to download Supplemental or Multimedia Files: Sala-Llonch_NBA_SuppMat.pdf Supplemental or Multimedia Files
Click here to download Supplemental or Multimedia Files: Sala-Llonch_NBA_SuppMat.pdf (1)

(n)
Click here to download Supplemental or Multimedia Files: Sala-Llonch_NBA_SuppMat.pdf

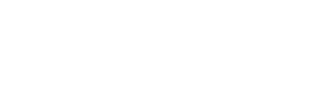
Click here to download Supplemental or Multimedia Files: Sala-Llonct

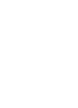

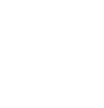

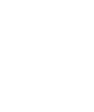

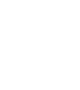

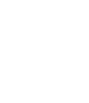


This work was supported by the Instituto Carlos III (PI12/00013 and PI16/00235 to RSV, cofunded by the European Regional Development Fund), by the Departament de Salut de la Generalitat de Catalunya (PERIS 2016-2020 SLT002/16/00329, to AA) and by an Investigator Sponsored Study founded by Life Molecular Imaging.

Life Molecular Imaging provided the FBB doses free of charge and funded the costs required by the Spanish Regulatory Agency (Investigator Sponsored Study (ISS) protocol code FBBFAD-2014; EudraCT No. 2014-000763-41).

The authors declare no competing interests. We thank all the volunteers for their participation in this study.

All authors had full access to the data in the study and the corresponding author had final responsibility for the decision to submit for publication.

Raquel Sanchez-Valle \& Roser Sala-Llonch on behalf of all authors. 


\section{HIGHLIGHTS}

- FBB-PET is safe in ADAD and useful to track amyloid progression.

- PSEN1 carriers showed progressive amyloid uptake.

- Amyloid uptake starts 15 years before onset in cortical areas and in the caudate.

- The rostral anterior cingulate can track disease progression in ADAD.

- Amyloid uptake coincides with increased cortical thickness. 